\title{
Preparation of Polyimide@ Polypyrrole/Palladium Hollow Composites with Applications in Catalysis
}

\author{
Xue FANG, Guiming SU *, Meihui SONG, Haijian JIANG, Xianghong CUI, \\ Xiaochen ZHANG, Mingyue CHEN
}

Institute of Advanced Technology, Heilongjiang Academy of Sciences, Harbin150020, China

crossref $h$ ttp://dx.doi.org/10.5755/j02.ms.23144

Received 01 April 2019; accepted 16 September 2019

\begin{abstract}
Polyimide based hollow composites were successfully prepared by combining the situ and template methods together. Firstly, the complete words (PPy) shell was covered on the surface of polystyrene nanoparticles, then the polyamide acid and $\mathrm{PdCl}_{2}$ were covered on the surface of the materials after the calcination. Finally, PI@ PPy/Pd hollow composites were obtained by a chemical process. The structure and composition of composites were characterized by scanning electron microscopy X-ray diffraction, thermo gravimetric analyzer and differential scanning calorimeter, respectively. Palladium (Pd) nanoparticles were densely and uniformly anchored on the surface of PPy shell due to the coordination interaction between amino groups on PPy backbone and $\mathrm{Pd}^{2+}$ ions. They are ideal candidates as nanoreactors for heterogeneous catalysis due to their special structure. The catalytic performance of PI@ PPy/Pd hollow composites were studied by the reduction of $\mathrm{NaBH}_{4}$ as a reducing agent. The catalytic performance of composites was characterized by ultraviolet and visible spectrophotometer. The PI@PPy/Pd composites showed excellent catalytic performance in the reduction of methylene blue with sodium borohydride as reducing agent. The dye completely turned colorless as seen within 2.0 minutes.

Keywords: polyimide, palladium nanoparticles, hollow composites, catalytic performance.
\end{abstract}

\section{INTRODUCTION}

With the gradual development of nanoparticles, researchers were focused on the structure of micro size more and more attentions [1]. Metal nanoparticles have drawn more and more attentions due to their special optical, electronic and catalytic properties originated from quantum size effects [2-5]. Because of their large surface area, metal nanoparticles have showed high catalytic activity in different type of reactions [6,7], the metal nanoparticles easily aggregate to large clusters due to their high surface energy, which may result in their deactivation [8]. Then it is a problem to solution for the accumulation of metal nanoparticles as a catalyst [9]. At present, the treatment of high temperature dye wastewater is still the model of cooled and then reprocessed $[10,11]$. It is very important that the economy of heat energy in the process [12].

Over the past decade, core/shell nanostructures have found their wide range of applications due to their optical, electrical, and catalytic properties. For hollow spheres with defined morphology and stability, specific molecular chains are required. Template synthesis based on core-shell structures has been well developed to achieve hollow spheres with different shell compositions. Hard cores such as silica colloid or polystyrene latex are commonly used as templates for coating various materials forming a coreshell structure, which are usually assisted by layer-by-layer (LBL) deposition. Shinji Watanabe used polystyrene as a template to prepare polystyrene-polyamic acid core/shell microspheres firstly, then polystyrene-polyimide core/shell

\footnotetext{
* Corresponding author. Tel.: 13624510365.

E-mail address: suguim@163.com (G. Su)
}

microspheres by a chemical process are obtained. It is used toluene to remove the core in order to obtain the hollow polyimide microspheres. Then the microspheres were chemically obtained, washed and dried, the pure polyimide hollow microspheres are obtained finally. However, the process of removing the core also caused the rupture of the polyimide shell.

Among the organic materials, polyimide (PI) is considered to be one of the promising materials, mainly because of its many advantages such as outstanding combination of thermal, mechanical and electrical insulating properties [13-16]. Among these, PI hollow nanospheres have generated significant interest in both fundamental and applied research, owing to their various potential applications such as low dielectric materials, high-temperature nanocontainers and confined reaction vessels with heat resistance. Jingjing used crosslinked sulfonated polystyrene microspheres as a template, then hollow polyimide microspheres are prepared. First, the linear portion of the sulfonated polystyrene spheres are removed, then dropped then solution of polyamic acid into the solution of the sulfonated spheres slowly, at the same time, with electromagnetic stirring, centrifugation and washing, sulfonated polystyrene-polyimide hollow microspheres are obtained. Finally, pyridine and acetic anhydride are added, the yellow microspheres are precipitated by a chemical process. Then the hollow microspheres were calcined at $350{ }^{\circ} \mathrm{C}$. It is increased the specific surface area and reduced the processing difficulty of polyimide materials for prepared into micro/nano spheres [17]. Therefore, the preparation and application of the composites based on polyimide have become a quickly expansive area [18-20]. 
In this paper, the polypyrrole (PPy) shell was covered on the surface of polystyrene nanoparticles, after calcination, the PPy hollow composites were obtained. We prepared hollow composites which coating polyamide acid on the surface, the composite materials were obtained and used as supports for deposition and adsorption of palladium (Pd) nanoparticles. Next, the polyimide @PPy/Pd composites were obtained. The polyimide @PPy/Pd composites showed excellent catalytic performance in the reduction of methylene blue with sodium borohydride as reducing agent.

\section{EXPERIMENTALS DETAILS}

The following materials were used in the experiments: Polystyrene nanoparticles (Jenus New Materials Co., Ltd.); Pyrrole monomer (Zhongguo Group Chemical Reagent Co., Ltd.); Palladium chloride (AR Sinopharm Chemical Reagent Co., Ltd.); N,N-dimethylacetamide (AR Tianjin Komi Chemical Reagent Co., Ltd.); Diaminodiphenyl ether (Zhongguo Group Chemical Reagent Co., Ltd.); Pyromellitic dianhydride (Zhongguo Group Chemical Reagent Co., Ltd.); Methylene blue(analytical grade and purchased from Zhongguo Group Chemical Reagent Co., Ltd.); $\mathrm{FeCl}_{3} \cdot 6 \mathrm{H}_{2} \mathrm{O}$ (analytical grade and purchased from Zhongguo Group Chemical Reagent Co., Ltd.); $\mathrm{NaBH}_{4}$ (analytical grade and purchased from Zhongguo Group Chemical Reagent Co., Ltd.); Absolute ethanol (Tianjin Yongda Chemical Reagent Co., Ltd.); The water used in the experiment is distilled water.

The structures of nanoparticles and nanocomposites were investigated by Lambda7600-FT-IR. A JEOL JSM-6700F scanning electron microscope (SEM) with primary electron energy of $3 \mathrm{keV}$ employed to examine the surface morphologies of products. Inorganic content of the composite spheres was measured by DSC-404 F3 and TG-209 F3. X-ray diffraction (XRD) data was collected on a X'Pert PRO X-ray diffractometer. New century general T6 ultraviolet and visible (UV-Vis) spectrophotometer was employed for analysis of MB dye reduction.

The PI@PPy/Pd nanocomposites were prepared by the following procedure. $5 \%$ solid content of PS nanoparticles $20 \mathrm{~mL}$ were dispersed in ethanol with mechanical stirring at room temperature. Then $1.2 \mathrm{~mL}$ pyrrole was added into the above solution with mechanical stirring. After 30 minutes, $10 \mathrm{~mL} \mathrm{FeCl} \cdot 6 \mathrm{H}_{2} \mathrm{O}(0.1 \mathrm{~g} / \mathrm{mL})$ was added into the mixed solution and dissolved completely, the mixture was reacted for $12 \mathrm{~h}$ continuously with mechanical stirring. $\mathrm{FeCl}_{3} \cdot 6 \mathrm{H}_{2} \mathrm{O}$ is an oxidizing agent. When the oxidizing agent was added into a mixed solution of pyrrole monomer and polystyrene, the pyrrole monomer was oxidatively polymerized in situ on the surface of the polystyrene microsphere. The precipitate was centrifuged and washed with deionized water and alcohol for three times. Then the precipitate was dried in oven under $60{ }^{\circ} \mathrm{C}$ for $12 \mathrm{~h}$. Then the PS@PPy nanocomposite were fired in muffle under $350{ }^{\circ} \mathrm{C}$ for $2 \mathrm{~h}$. The PPy hollow microspheres were obtained.

$40 \mathrm{~g}$ 4,4'-oxydianiline (ODA) was added into the N,N'-dimethylacetamide (DMAc) $(500 \mathrm{~mL})$, then $62 \mathrm{~g}$ pyromelliticdianhydride (PMDA) in stoichiometric amount to ODA was added by portions, the mixture was reacted for about $12 \mathrm{~h}$ continuously with agitation at room temperature. Then the PAA was obtained.

$80 \mathrm{mg}$ PPy hollow microspheres were dispersed in $50 \mathrm{~mL}$ DMAc with mechanical stirring, then PAA $320 \mathrm{mg}$ of $\mathrm{PdCl}_{2}$ was added in the system with mechanical stirring for $24 \mathrm{~h}$. The precipitate was centrifuged and washed with DMAc for three times. Then the precipitate was dried in oven for $12 \mathrm{~h}$.

40 mg PAA@PPy/Pd nanocomposites were dispersed in $50 \mathrm{~mL}$ DMAc with mechanical stirring, then $1 \mathrm{~mL}$ pyridine and $2 \mathrm{~mL}$ acetic oxide were added in the system, the reaction was allowed to proceed with stirring for $8 \mathrm{~h}$ at $60{ }^{\circ} \mathrm{C}$. Then the nanocomposites were allowed to stand for $24 \mathrm{~h}$. After washed with deionized water and ethanol several times, the nanocomposites were dried in oven for 6 h. Then the PI@PPy/Pd nanocomposites were obtained.

\section{RESULTS AND DISCUSSION}

The whole procedure to prepare PI@PPy/Pd composites is illustrated in Fig. 1.

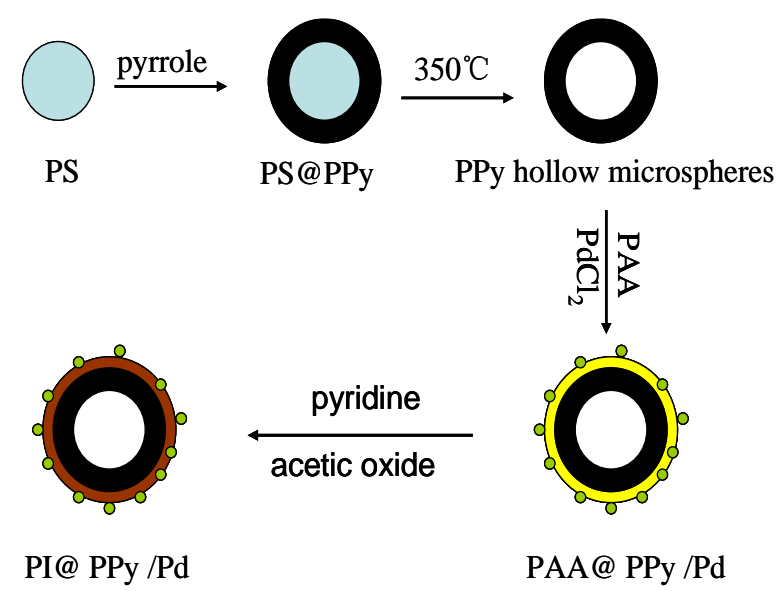

Fig. 1. Synthesis route to PI@PPy/Pd composites

The equations of chemical reactions that occurred during the synthesis are illustrated in Eq. 1 and Eq. 2.

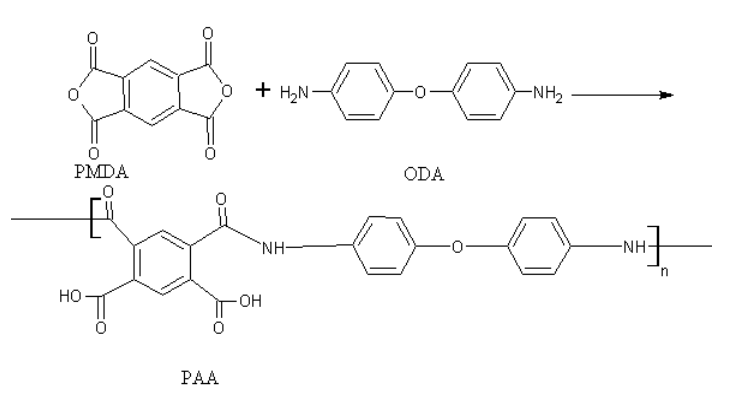

Fig. 2 shows the SEM micrographs of PS nanoparticles, PS@PPy nanocomposites, PPy hollow microspheres, and PI@PPy/Pd composites. Fig. 2 a shows the PS nanoparticles have smooth surface and narrow size distribution, which are suitable for templates. The morphology of PS@PPy nanocomposites is presented in Fig. 2 b, compared with PS composites, their surface becomes much rougher, indicating polymerization of pyrrole monomer has occurred. Fig. $2 \mathrm{c}$ shows the PPy hollow microspheres, the nanocomposites have smooth 
again and the ruptured hollow composites indicate that the PPy hollow microspheres are of hollow structures. In Fig. 2 d, it can be seen that the Pd nanoparticles deposit on the supports.

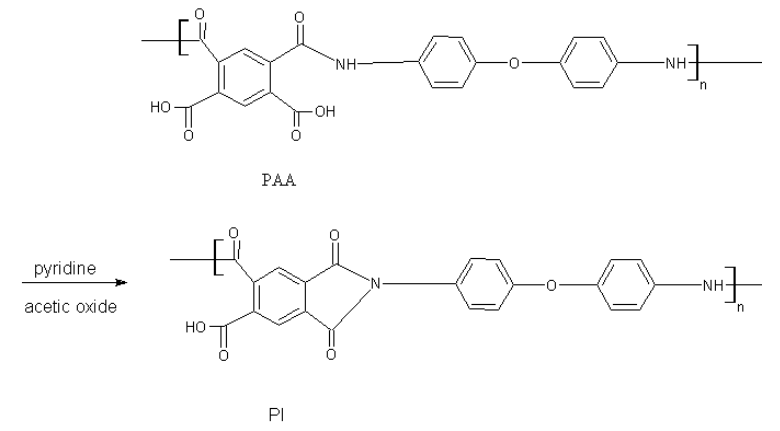

(2)

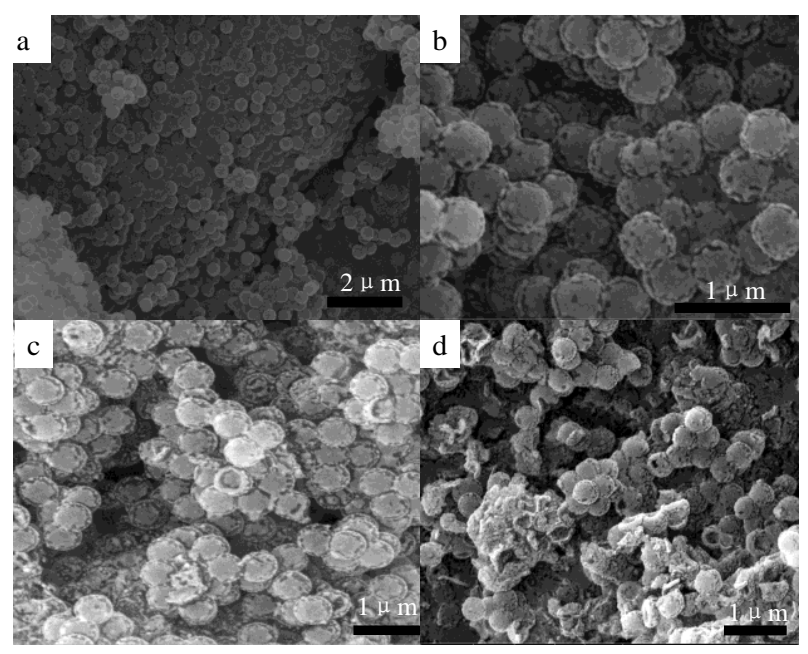

Fig. 2. SEM images: a-PS nanoparticles; b-PS@PPy nanocomposites; c-PPy hollow microspheres; dPI@PPy/Pd composites

Fig. 3 shows the FT-IR spectrum of the samples. In Fig. 3a the spectra displayed the characteristic features of PS nanoparticles. The main absorption peaks of PS nanoparticles appeared in 3026, $2923 \mathrm{~cm}^{-1}$ are assigned to C-H stretching vibrations of olefins [9]. The peaks at 1492 and $1448 \mathrm{~cm}^{-1}$ are assigned to $\mathrm{C}-\mathrm{H}$ bending vibration in chains. And the peek at $699 \mathrm{~cm}^{-1}$ is assigned to $\mathrm{C}-\mathrm{H}$ skeleton vibration and benzene ring skeleton vibration [9].

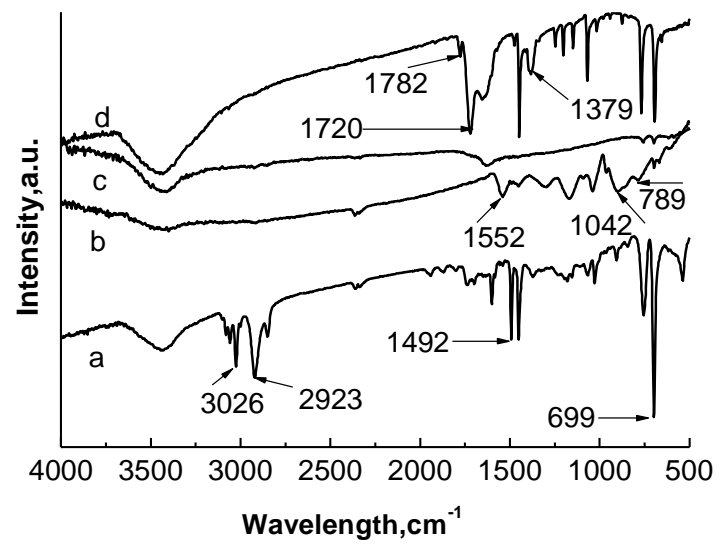

Fig. 3. FT-IR spectra: a-PS nanoparticles; b-PS@PPy nanocomposites; c-PPy hollow microspheres; d-PI@PPy/Pd hollow composites
In Fig. $3 \mathrm{~b}$ the spectra displayed the characteristic features of PPy. The peaks at 1552 and $1042 \mathrm{~cm}^{-1}$ are due to the stretching mode of $\mathrm{C}-\mathrm{H}$ bonds, and the absorption peak of polystyrene occurred at $789 \mathrm{~cm}^{-1}$. A part of absorption peak of PS microspheres appeared due to the coating of PPy. In Fig. $3 \mathrm{c}$ the absorption peaks of PS and PPy all disappeared, it was illustrated that the PS microspheres have been decomposed and the PPy shells have been carbonized, leaving only the carbon skeleton structure. In Fig. $3 \mathrm{~d}$ the spectra displayed the characteristic features of $\mathrm{PI} @ \mathrm{PPy} / \mathrm{Pd}$ composites, the peaks at 1782 and $1720 \mathrm{~cm}^{-1}$ are due to the stretching mode of $\mathrm{C}-\mathrm{O}$ bonds, and the peak at 1379 is due to the stretching mode of $\mathrm{C}-\mathrm{N}$ bonds. Among them, the peaks at $3400 \mathrm{~cm}^{-1}$ is assigned to $\mathrm{O}-\mathrm{H}$ vibration. The presence of $\mathrm{O}-\mathrm{H}$ bond is due to the absorption of moisture by the material. In Fig. $3 \mathrm{~d}$, the peak at $3400 \mathrm{~cm}^{-1}$ is due to acetic acid was added during the preparation process, and acetic acid could not be completely dried. It was illustrated that the PI was existed in the PI@PPy/Pd hollow composites.

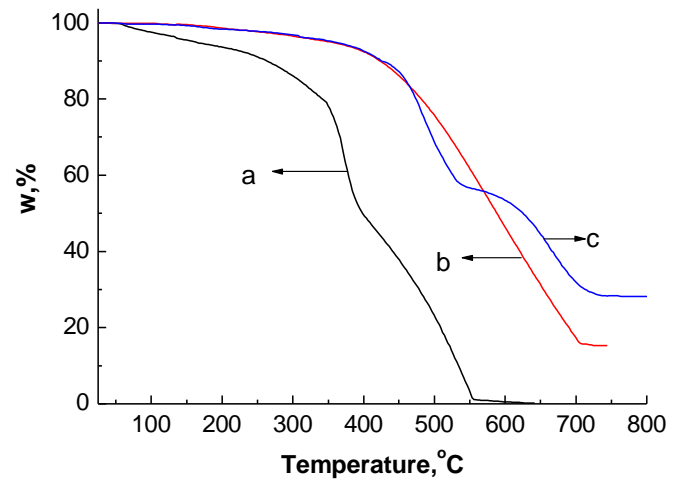

Fig. 4. TGA spectra: a-PS@PPy nanocomposites; b-PPy hollow microspheres; c-PI@PPy/Pd hollow composites

The samples were subjected to thermal stability analysis by a thermogravimetric analyzer, and the test environment were air (Fig. 4 a), $\mathrm{N}_{2}$ (Fig. 4 b), and atmosphere (Fig. 4 c). Fig. 4 shows the thermogravimetric curves. As can be seen from the curve a, the weight is quickly lost at around $300^{\circ} \mathrm{C}$ and finally completely decomposed under an air atmosphere. The initial weight loss at $450{ }^{\circ} \mathrm{C}$ was the polypyrrole hollow material of curve b. This is because polypyrrole was not easily degraded in oxygen-free nitrogen, and the presence of oxygen in the air promoted its degradation. Therefore, we conducted a thermal weight loss test under a nitrogen atmosphere, and a part of the composite was burned off, leaved only a part of the polypyrrole skeleton which was not easily decomposed. Curve $\mathrm{c}$ shows two sections of thermogravimetric curves at $450{ }^{\circ} \mathrm{C}$ and $550{ }^{\circ} \mathrm{C}$. This is due to the different thermal decomposition temperatures of polyimide and polypyrrole, and the metal palladium as an inorganic portion and a part of the polypyrrole skeleton were left. By calculated the data of curves $b$ and $c$, it was possible to obtain a residual of metal palladium of up to $15 \%$ or more.

Fig. 5 shows the DSC curves. The exothermic peak at $600{ }^{\circ} \mathrm{C}$ (curve a) was the thermodynamic behavior of PPy hollow microspheres. And the exothermic behavior begins at $500{ }^{\circ} \mathrm{C}$, this is due to the fact that only the polypyrrole 
skeleton remains after calcination. Curve $\mathrm{b}$ shows two exothermic peaks at $300{ }^{\circ} \mathrm{C}$ and $550{ }^{\circ} \mathrm{C}$, this is the thermal decomposition behavior of PS and PPy. Curve $\mathrm{c}$ shows two exothermic peaks at $300^{\circ} \mathrm{C}$ and $550{ }^{\circ} \mathrm{C}$, this is the thermal decomposition behavior of PI and PPy. This is due to the different thermal decomposition temperatures of PI and Ppy.

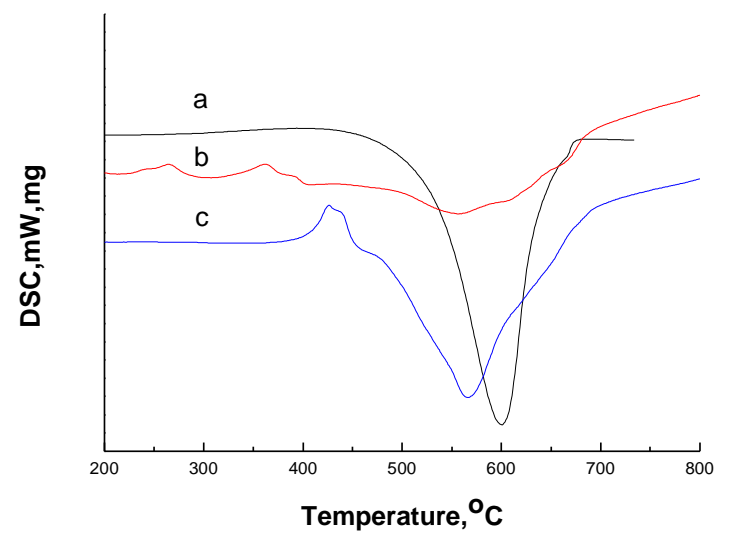

Fig. 5. DSC spectra: a-PPy hollow microspheres; b-PS@PPy nanocomposites; c-PI@ PPy /Pd hollow composites

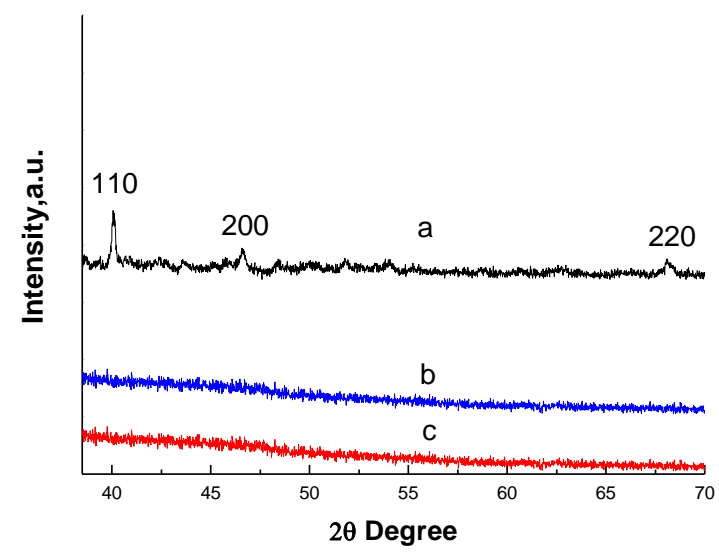

Fig. 6. XRD patterns: a-PI@PPy/Pd hollow composites; b-PPy hollow microspheres; c-PS@PPy nanocomposites

XRD patterns are presented in Fig. 6. The crystal structure of the palladium nanoparticles was confirmed and the diameter was calculated by a formula. We can see in the figure that the PI@PPy/Pd hollow composites exhibited strong diffraction peaks at $2 \theta=40.1^{\circ}, 46.6^{\circ}$ and $68.1^{\circ}$, which are corresponding to the crystal plane structures of (110), (200) and (220) of the palladium nanoparticles respectively (JCPDS No.04-0784), there are no diffraction peaks in curve $b$ and curve $c$, it confirmed that the particles we synthesized were palladium nanoparticles. The average diameter of the palladium nanoparticles was calculated according to the DebyeScherrere formula [21].

Taking the diffraction peak at $2 \theta=68.1^{\circ}$ as a reference, the size of the palladium nanoparticles was calculated using the Debye-Scherrere formula (Eq. 3), and the average diameter of the obtained palladium nanoparticles was estimated to be $10.3 \mathrm{~nm}$. Among this numerical values, $K$ is the Scherrer constant, the numerical value is $0.89, \lambda$ is the $\mathrm{X}$-ray wavelength, and the numerical value is $0.15418 \mathrm{~nm}$.

$$
D=\frac{k \lambda}{B \cos \theta},
$$

where $B$ is the half width; $\theta$ is the diffraction angle, ${ }^{\circ} ; \lambda$ is the wavelength of $\mathrm{X}$-rays, $\mathrm{nm} ; K$ is the calculation constant.

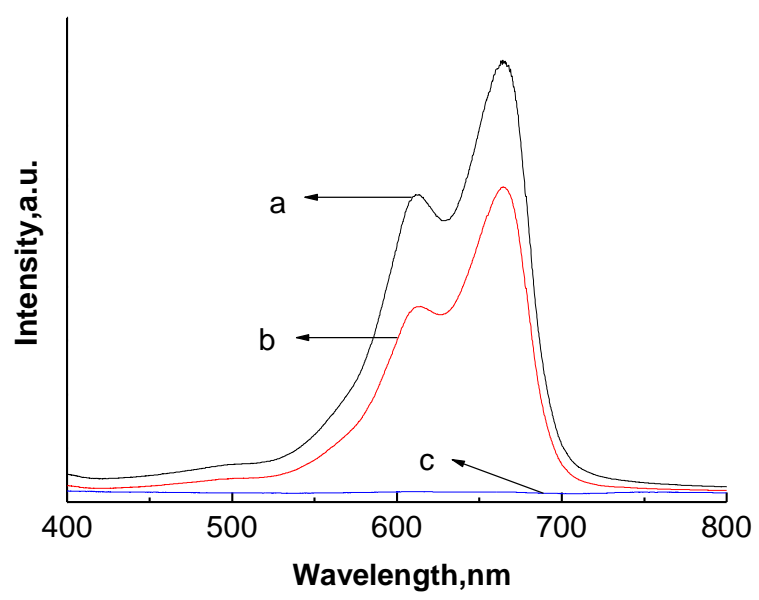

Fig. 6. Vis spectra: a-the initial methylene blue dye solution; $\mathrm{b}$-the methylene blue dye solution after reaction for $8 \mathrm{~h}$ without PI@PPy/Pd hollow composites; c-the methylene blue dye solution after reaction for $2 \mathrm{~min}$ in presence of PI@PPy/Pd hollow composites

The reduction of methylene blue (MB) dye by $\mathrm{NaBH}_{4}$ was selected as a model reaction to study the catalytic activity of the PI@PPy/Pd composites. We prepared $(0.2 \mathrm{mg} / \mathrm{mL})$ aqueous solution of MB $3.5 \mathrm{~mL}$. The reaction process was monitored by Vis spectra, as illustrated in Fig. 4 a. The original aqueous MB dye solution is dark blue in color and the kmax appears at $665 \mathrm{~nm}$ (curve a). $2 \mathrm{~mL}$ sodium borohydride solution $\left(\mathrm{NaBH}_{4}\right)(10 \mathrm{mg} / \mathrm{mL})$ was added to the system.

Upon the addition of $\mathrm{NaBH}_{4}$ without catalysts, the absorbance intensity at kmax of MB decreases, but does not completely vanish after reaction for $8.0 \mathrm{~h}$ (curve b). The solution color only becomes light blue. After $10.0 \mathrm{mg}$ of the PI@ PPy/Pd composites were added, the absorbance at kmax completely disappears within 2.0 minutes (curve c). The solution color turns colorless as seen. It could be concluded that the PI@PPy/Pd composites had excellent catalytic performance in reduction of $\mathrm{MB}$ dye with $\mathrm{NaBH}_{4}$ as a reducing agent.

\section{CONCLUSIONS}

In this article, we have successfully synthesized PI@ PPy/Pd hollow composites. Firstly, the PPy shell was covered on the surface of PS nanoparticles, then the PAA and $\mathrm{PdCl}_{2}$ were covered on the surface of the material that after the calcination. Finally, PI@PPy/Pd hollow composites were obtained by a chemical process. They are ideal candidates as nanoreactors for heterogeneous catalysis due to their special structure. And the results showed that the PI@PPy/Pd hollow composites could keep excellent spherical structure, and the PI@PPy/Pd hollow composites showed excellent catalytic performance in the reduction of methylene blue with sodium borohydride as reducing agent. The dye completely turned colorless as 
seen within 2.0 minutes. This method not only simplifies the process conditions of the conventional method, but also solves the problem of poor adhesion between metal and polymer. The biggest advantage of this method is that the doped nano-palladium can be distributed in the polymer system with the original particles, so as to best reflect or maintain the unique properties of the nano-palladium. More novelly, due to the special properties of polyimide, this hollow composite can handle high temperature wastewater and special wastewater containing acid and alkali. Therefore it is anticipated that this kind of catalysts will have great potential for further practical applications.

\section{REFERENCES}

1. Yao, T.J., Cui, T.Y., Fang, X., Yu, J., Cui, F., Wu, J. Preparation of Yolk/shell $\mathrm{Fe}_{3} \mathrm{O}_{4} @$ polypyrrole Composites and Their Applications as Catalyst Supports Chemical Engineering Journal 225 2013: pp. 230-236. https://doi.org/10.1016/j.cej.2013.02.026

2. Murray, R.W. Nanoelectrochemistry: Metal Nanoparticles, Nanoelectrodes, and Nanopores Journal of Chemical Research 108(11) 2008: pp. 2688-2720.

http://link.springer.com/article/10.1007\%2Fs115950110163-y

3. Crooks, R.M., Zhao, M.Q., Sun, L., Chechik, L., Yeung, L.K. Dendrimer-encapsulated Metal Nanoparticles: Synthesis, Characterization, and Applications to Catalysis Journal of Chemical Research 34(3) 2001: pp. 181-190. https://doi.org/10.1002/(SICI)15214095(199903)11:33.0.CO ;2-7

4. Jain, P.K., Lee, K.S., El-Sayed, I.H., El-Sayed, M.A. Calculated Absorption and Scattering Properties of Gold Nanoparticles of Different Size, Shape, and Composition: Applications in Biological Imaging and Biomedicine Journal of Physical Chemistry B 110 (14) 2006: pp. 7238-7248. https://doi.org/10.1021/jp057170o

5. Han, S.Y., Guo, Q.H., Xu, M.M., Yuan, Y.X., Shen, L.M., Yao, J.L., Liu, W., Gu, R.A. Tunable Fabrication on Iron Oxide/Au/Ag Nanostructures for Surface Enhanced Raman Spectroscopy and Magnetic Enrichment Journal of Colloid and Interface Science 378 (1) 2012: pp. 51-57. https://doi.org/10.1016/j.jcis.2012.04.047

6. Tournebize, J., $\quad$ Boudier, A., $\quad$ Sapin-Minet, A., Maincent, P., Leroy, P., Schneider, R. Role of Gold Nanoparticles Capping Density on Stability and Surface Reactivity to Design Drug Delivery Platforms ACS Applied Materials \& Interfaces 4 2012: pp. 5790-5799. https://doi.org/10.1021/am3012752

7. Leung, K.C.F., Xuan, S.H., Zhu, X.M., Wang, D.W., Chak, C.P., Lee, S.F., Ho, W.K.W., Chung, B.C.T. Gold and Iron Oxide Hybrid Nanocomposite Materials Chemical Society Reviews 41 2012: pp. 1911-1928. https://doi.org/10.1002/chin.201220217

8. Lee, J., Park, J.C., Song, H. A Nanoreactor Framework of a Au@ $\mathrm{SiO}_{2}$ Yolk/Shell Structure for Catalytic Reduction of p-Nitrophenol Advanced Materials 20 2008: pp. $1523-1528$.

https://doi.org/10.1002/adma.200702338

9. Yao, T.J., Cui, T.Y., Fang, X., Cui, F., Wu, J. Preparation of Gold Nanorods@C Hollow Capsules and Applications in
Catalysis Chemical Journal of Chinese Universities 34 (10) 2013: pp. 2421-2426.

https://doi.org/10.1016/j.cej.2013.02.026

10. He, J., Jing, J., Zhao, X.G., Wang, W., Liu, C.W., Zhou, H.W. Preparation and Characterization of Polyimide Magnetic Hollow Nanospheres Materials Letters 2012: pp. $86-89$. https://doi.org/10.1016/j.matlet.2011.10.004

11. Hao, O.J., Jimand, H., Chiang, P.C. Decolorization of Wastewater Critical Reviews in Environmental Science and Technology 30 (4) 2000: pp. 449-505. https://doi.org/10.1080/10643389991259245

12. Xue, H.W., Zhao, S.X. Thermophiles and Their Uses for Waste Treatment Journal of Tianjin University of Light Industry 9 (3) 2002: pp. 7-10. https://doi.org/10.1016/j.matdes.2010.07.018

13. Liu, Q.P., Gao, L.X., Gao, Z.W., Yang, L. Preparation and Characterization of Polyimide/silica Nanocomposite Spheres Materials Letters 61 2007: pp. 4456-4458. https://doi.org/10.1002/pc.20372

14. Chang, C.C., Chen, W.C. Synthesis and Optical Properties of Polyimide-Silica Hybrid Thin Films Chemistry of Materials 14 2002: pp. $42-42$. https://doi.org/10.1016/j.matchemphys.2008.08.012

15. Musto, P., Ragosta, G., Scarinzi, G., Mascia, L. Polyimide-silica Nanocomposites: Spectroscopic, Morphological and Mechanical Investigations Polymer 45 2004: pp. $1697-1700$. https://doi.org/10.1016/j.polymer.2003.12.044

16. Mascia, L., Kioul, A. Influence of Siloxane Composition and Morphology on Properties of Polyimide-silica Hybrids Polymer 36 1995: pp. 3649-3652. https://doi.org/10.1016/0032-3861(95)93766-f

17. Lu, J.J., Chen, L., Liu, M.Q., Yan, Y.D., Zhao, X.B. Preparation of Poly(amic acid) Particles by Precipition Polymerization and Study on the Imidization of poly(amic acid) Journal of Shanxi Chemical Industry 31 (4) 2011: pp. $18-23$. https://doi.org/10.3724/SP.J.1105.2010.10073

18. Zhang, Y., Jiang, L., Li, H., Fan, L.Z, Hu, W.P., Wang, C.R., Li, Y.F., Yang, S.H. Single-crystalline C60 Nanostructures by Sonophysical Preparation: Tuning Hollow Nanobowls as Catalyst Supports for Methanol Oxidation Chemistry-A European Journal 17 2011: pp. 4921-4926. https://doi.org/10.1002/chem.201002719

19. Yu, X.L., Liang, F.X., Liu, J.G., Lu, Y.F., Yang, Z.Z. Simultaneous Adsorption of Atrazine and $\mathrm{Cu}$ (II) from Wastewater by Magnetic Multi-walled Carbon Nanotube Journal of Colloid and Interface Science 367 2012: pp. $531-536$ https://doi.org/10.1016/j.cej.2012.09.102

20. Li, Q., Yang, X., Chen, W., Yi, Ch., Xu, Z. Preparation of Poly(amic acid) and Polyimide via Microwave-Assisted Polycondensation of Aromatic Dianhydrides and Diamines Macromolecular Symposia 261(1) 2008: pp. 148-156. https://doi.org/10.1002/masy.200850120

21. Todorov, R., Lozanova, V., Knotek, P., Cernoskova, E., Vicek, M. Microstructure and Ellipsometric Modelling of the Optical Properties of Very Thin Silver Films for Application in Plasmonics Thin Solid Films $628(30)$ 2017: pp. $22-30$. https://doi.org/10.1016/j.tsf.2017.03.009

(c) Fang et al. 2021 Open Access This article is distributed under the terms of the Creative Commons Attribution 4.0 International License (http://creativecommons.org/licenses/by/4.0/), which permits unrestricted use, distribution, and reproduction in any medium, provided you give appropriate credit to the original author(s) and the source, provide a link to the Creative Commons license, and indicate if changes were made. 